\title{
Using marginal structural models to identify the cardiovascular adverse effects of second generation antipsychotics in children and adolescents
}

\author{
Avnish Tripathi ${ }^{1}$, George B. Black ${ }^{2}$, Jeanette M. Jerrell ${ }^{* 3}$ \\ ${ }^{1}$ Department of Medicine, University of Louisville School of Medicine, Louisville, KY USA \\ ${ }^{2}$ Department of Internal Medicine, University of Virginia School of Medicine, Charlottesville, VA USA \\ ${ }^{3}$ Department of Neuropsychiatry and Behavioral Science, University of South Carolina School of Medicine, Columbia, SC USA
}

Received: May 25, 2015

DOI: $10.5430 /$ jer.v1n1p5
Accepted: June 28, 2015

Online Published: July 14, 2015

\begin{abstract}
In pediatric patients, we examined the association between exposure to five second generation antipsychotics (SGAs) and incident cardiovascular events (arrhythmic or ischemic/myocardial) over time using marginal structural models (MSM), while controlling for salient comorbid conditions and co-prescribed psychotropic medications. A retrospective cohort, longitudinal/observational study design was used to evaluate Medicaid medical and pharmacy claims in 4,140 children and adolescents prescribed SGAs from South Carolina USA's Medicaid program covering outpatient and inpatient medical services and medication prescriptions between January, 1996 and December, 2005. Exposure to multiple SGAs (Risk Ratio [RR]=2.37; 95\% CI=1.17-4.83), coprescribed psychostimulants $(\mathrm{RR}=1.37 ; \mathrm{CI}=1.03-1.81)$, and comorbid hypertension $(\mathrm{RR}=2.23 ; \mathrm{CI}=1.28-3.89)$ were associated with a significantly increased risk of arrhythmias compared to those not exposed, whereas exposure to co-prescribed serotonin norepinephrine reuptake inhibitor/heterocyclic compounds was associated with a significantly decreased risk of arrhythmias $(\mathrm{RR}=0.59 ; \mathrm{CI}=0.35-0.99)$. The risk of incident ischemic/myocardial events was significantly associated with the co-prescription of mood stabilizers $(\mathrm{RR}=1.68 ; \mathrm{CI}=1.06-2.68)$ or selective serotonin reuptake inhibitors $(\mathrm{RR}=1.91 ; \mathrm{CI}=1.18-3.09)$, and the presence of comorbid hypertension ( $R R=3.97 ; C I=1.96-8.07)$ and obesity $(R R=2.21 ; C I=1.34-3.67)$. MSM analyses comparing multiple treatments while controlling for confounding variables in an observational, longitudinal data set provide important, differential estimates of outcome, when randomized, controlled trials estimating low-incidence outcomes such as cardiovascular adverse events in large pediatric patient populations are not feasible.
\end{abstract}

Key Words: Children, Adolescents, Antipsychotics, Marginal structural models, Pharmaco-epidemiology, Adverse events

\section{INTRODUCTION}

Investigators have monitored the substantial increase in the use of second generation antipsychotic medications (SGAs) in child and adolescent patients for more than a decade. ${ }^{[1-4]}$ Based on more recent controlled trials and observational studies, cardiovascular safety concerns associated with the use of SGAs in younger patients include tachycardia, QTc prolongation and other arrhythmias, myocarditis, and sudden cardiac death. ${ }^{[1,5,6]}$ Moreover, SGAs are associated with clinically significant weight gain and alterations in metabolic

\footnotetext{
* Correspondence: Jeanette M. Jerrell, Ph.D; Email: Jeanette.Jerrell@uscmed.sc.edu; Address: Department of Neuropsychiatry and Behavioral Science, 3555 Harden Street Ext., Suite 301, Columbia, SC 29203, USA. 
indices (e.g., type 2 diabetes mellitus, dyslipidemia) in young populations, especially adolescents, which in turn may be associated with the earlier onset of cardiovascular disease in adulthood ${ }^{[7,8]}$ Similar cardiovascular events have also been associated with other classes of psychotropic agents frequently co-prescribed with antipsychotics in younger populations, such as several selective serotonin reuptake inhibitors (SSRIs), serotonin norepinephrine reuptake inhibitor/ heterocyclic compounds (SNRIs), ${ }^{[9-11]}$ psychostimulants, ${ }^{[12-14]}$ and mood stabilizers. ${ }^{[7,15]}$ However, cardiovascular disorders have never been linked directly to the underlying psychiatric conditions.

Managing these myriad potential risks in clinical practice requires specific estimates of the relative contribution of each risk factor. Such studies have not been available because cardiovascular events are rare in children, except in those with congenital heart disorders or chromosomal syndromes, and these investigations require very large numbers of representative patients. Major barriers to conducting informative, randomized controlled trials (RCTs) evaluating cardiovascular safety concerns across multiple SGAs and co-prescribed psychotropic medications relate to cost, ethics, and identifying large numbers of representative subjects. Alternatively, longitudinal observational data sets are more representative, readily available, and may complement results from RCTs. However, such studies are vulnerable to confounding and some loss of internal validity. ${ }^{[16]}$ Estimation of the causal effect of an exposure on an outcome may be biased because of time-varying confounders, where exposure to each treatment (e.g., antipsychotic, other psychotropic agents, medication switches, etc.) varies over time, the development of potential adverse responses (e.g., adverse events or comorbid conditions) also varies over time, and the observed response differences may not be attributed directly to a particular treatment exposure. ${ }^{[17]}$ In observational studies, marginal structural modeling (MSM) allows for more precise estimation of treatment effects by providing statistical controls for potentially confounding conditions or treatments, and for selection bias. ${ }^{[18-21]}$ Furthermore, MSM techniques can be used in analyzing multiple treatment effects and adjusting for treatment group differences in observational studies through the use of time-dependent inverse-probability treatment weights. ${ }^{[16,22,23]}$

In this re-analysis of a longitudinal observational data set, we sought to demonstrate the added value of using MSM by comparing the cumulative incidence of various cardiovascular events across SGA agents in an SGA-treated cohort of one USA state's Medicaid system, controlling for salient confounders such as co-prescribed psychotropic medications and comorbid metabolic disorders.

\section{MAterials AND MethodS}

\subsection{Study cohort, medications, and outcomes}

Access to healthcare in South Carolina (SC) USA is funded predominantly by public (for low-income families) or private insurance payers, which cover over $99 \%$ of the children and adolescents residing in the state. Once a child is diagnosed with a serious or disabling condition, including psychiatric disorders, the family can apply for Medicaid coverage of his/her special medical needs regardless of income.

Medical and pharmacy claims for the calendar years January 1, 1996 through December 31, 2005 were used to identify a cohort of child and adolescent patients ages 17 and under enrolled in and eligible for Medicaid coverage for a minimum of 9 months in each calendar year included in this analysis, who had a service encounter, and who were prescribed any of five SGAs (i.e. aripiprazole, ziprasidone, quetiapine, risperidone, olanzapine) being used in routine practice during this epoch. For each service encounter, the date of service and the International Classification of Diseases (ICD) Ninth Revision Clinical Modification diagnosis codes related to that visit were obtained. Pharmacy claims identified the medication dispensed, and the date the prescription was filled. A separate eligibility file was used to obtain the demographics for each patient served. For each patient in the data set, 24 months of services prior to the start date of the antipsychotic medication were captured to identify pre-existing or comorbid cardiovascular or metabolic conditions and to serve as the "no-exposure to antipsychotic medications" baseline period in the MSM analyses. These Medicaid databases are frequently updated and cleaned prior to being made available for research analysis. This study was approved by the University of South Carolina Institutional Review Board as exempt from human subject research guidelines under 45 Code of Federal Regulations part 46.

Since acquired cardiovascular events/conditions are relatively rare in children, individual cardiovascular events using the diagnostic codes reported for each service visit were combined into two primary outcome variable categories for these analyses: cumulative incidence of ischemic/myocardial events and cumulative incidence of cardiac conduction/arrhythmia events. Ischemic/myocardial events were defined as the reporting of at least one of the following ICD-9 codes: 410.xx-414.xx (ischemic heart disease); 422.xx (myocarditis); 428.xx-429.xx (heart failure/other conditions); or 425.xx (cardiomyopathy). Cardiac conduction disorders/arrhythmias were defined as the reporting of at least two ICD-9 codes (426.xx-427.xx) at least 30 days apart, but the first reported code was used as the index event.

The following categories of comorbid medical conditions 
and co-prescribed psychotropic medications were also coded and controlled for in the analyses because they have been previously associated with cardiovascular events. The timedependent (coded as a yes/no occurrence of each condition for each person-month of the study), comorbid medical conditions included: obesity/overweight $(278.00 ; 278.01 ; 783.1 \mathrm{x}$, 783.2x), dyslipidemia (272.xx, 288.0x, 285.9x), either Type 1 or Type 2 diabetes mellitus (250.00-251.92), essential hypertension (401.xx), congenital heart defects (747.0x-747.9x), cerebrovascular disorders (436.xx-437.xx), or a substancerelated disorder (304.xx and 305.xx). Each covariate medical condition was defined by the reporting of at least two visits with that diagnostic code that were at least 30 days apart to mitigate the risk of misclassification due to erroneous coding. Prescription medications were also coded as time-dependent covariates, i.e., their use might change over time. Co-prescribed antidepressants were categorized as SSRIs for citalopram, escitalopram, fluoxetine, fluvoxamine, paroxetine, and sertraline, or as SNRIs/heterocyclics/ others for duloxetine $\mathrm{HCl}$, mirtazapine, bupropion $\mathrm{HCl}$, venlafaxine $\mathrm{HCl}$, trazadone, and nefazodone. Mood stabilizers coded in the regression equations were divalproex, lithium, carbamazepine, lamotrigine, and oxcarbazepine. Psychostimulants coded in the analyses were methylphenidate, dextroamphetamine, and amphetamine salts. Co-prescribed first generation antipsychotics haloperidol or fluphenazine given orally or as intramuscular injections for acutely psychotic or aggressive behavior were also used as covariates in the analyses. The time independent (fixed) covariates included: age at entry into the cohort, gender, and race/ethnicity. These diagnoses and pharmacotherapies contained in the Medicaid billing system have been compared with information available in the clinical records of 300-400 children treated with antipsychotics or other psychotropic medications to provide validation of the secondary source data ${ }^{[24,25]}$

\subsection{Statistical analysis}

First, the stabilized, inverse-propensity treatment weights were calculated to estimate the probability of receiving each SGA depending on previous exposure history to no SGA during the 2-year baseline period or to other SGAs during the treatment period and the probability of censoring (i.e., leaving the data set) during the study treatment observation period, and to balance the treatment groups with respect to baseline confounders. ${ }^{[16,17,22]}$ Multivariable logistic regression was used to calculate these weights ${ }^{[26]}$ and we verified that the mean of these weights was close to $1.0 .^{[21]}$

In the second step, two weighted Cox proportional hazard MSM models were created using each cumulative incident ischemic/myocardial or arrhythmia event as the dependent

Published by Sciedu Press variable and time-dependent exposure to each SGA or no SGA in each person-month (dichotomized as a yes/no occurrence) as a predictor variable. Each weighted Cox proportional hazard MSM model also controlled for other timedependent covariates, including exposure to SSRIs, SNRIs, mood stabilizers, psychostimulants, and first generation antipsychotics (haloperidol or fluphenazine), the diagnosis of comorbid conditions of congenital heart defect, hypertension, obesity/overweight, diabetes mellitus, dyslipidemia, and substance-related disorders, and for time-independent individual risk factors of gender, race/ethnicity and age-group at study entry. This design enabled us to estimate the probability of incident cardiovascular events occurring in relation to whether or not a person received an SGA or a particular SGA during each month of the study period after controlling for simultaneous exposure to other SGAs or psychotropic medications, and related comorbid conditions. A critical assumption of MSM is that the probability of treatment must be nonzero, so the use of a no-treatment group in this analysis would substantially bias the results, ${ }^{[23]}$ and, therefore, was not warranted. Results comparing incident outcomes between the cohort exposed to antipsychotic treatment and a notreatment control group have been presented elsewhere. ${ }^{[27]}$ In the multivariable Cox proportional hazards models, interaction terms with a time variable were included if the proportional hazards condition was not met.

The measure of association reported is the adjusted rate ratio (RR) with a corresponding $95 \%$ confidence interval. $P$ values of less than 0.05 (two-sided tests) were considered statistically significant, and all statistical analyses were performed in SAS software, version 9.2 (SAS Institute, Cary, North Carolina).

\section{Results}

Characteristics of the cohort of 4140 children are presented in Table 1. Ischemic/myocardial events identified in these children were: ischemic heart disease in $1.3 \%$, heart failure in $2.7 \%$, cardiomyopathy in $0.2 \%$. Conduction/arrhythmia events were identified in $7.5 \%$ of the cohort. The prevalence of all covariates are also presented. During the study period, 12 individuals who died had a diagnosed cardiovascular condition (i.e., myocarditis and cardiomyopathy) after initiation of an antipsychotic and a cardio- or cerebrovascular condition was noted as their cause of death. However, because these numbers were very small and we could not directly associate the incident cardiovascular event with death using this dataset, only the incident cardiovascular condition was included in the MSM analyses. Moreover, significantly more individuals who died also had brain damage or severe mental retardation $\left(\mathrm{n}=15 ; \chi^{2}=32.58 ; p<.0001\right)$ or a seizure 
disorder $\left(\mathrm{n}=13 ; \chi^{2}=11.89 ; p=.0006\right)$, but significantly fewer were taking concomitant SSRIs $\left(\mathrm{n}=7 ; \chi^{2}=8.74 ; p=\right.$ $.003)$, or psychostimulants $\left(\mathrm{n}=11 ; \chi^{2}=14.87 ; p=.0001\right)$ than the children who did not die.

Table 1. Descriptive Analysis of the Cohort of 4140 Youths Prescribed Antipsychotic Medications

\begin{tabular}{ll}
\hline Indicator & N (\%) \\
\hline Gender: Male & $2825(68.2)$ \\
Race: Caucasian & $1722(41.6)$ \\
African American & $1680(40.6)$ \\
Other non-white (Hispanic, Asian, Unknown, & $738(17.8)$ \\
Mixed) & 10.4 years (SD: \pm 3.6$)$ \\
Mean Age at Start of Antipsychotic & $418(10.1)$ \\
Cumulative Cardiovascular Events/Disorders & $25(0.6)$ \\
Died during study period & \\
Comorbid Medical Conditions & $839(20.3)$ \\
Overweight/Obesity & $210(5.1)$ \\
Type 1 or Type 2 Diabetes Mellitus & $175(4.2)$ \\
Dyslipidemia & $290(7.0)$ \\
Primary Hypertension & $146(3.5)$ \\
Congenital Heart Defects & $91(2.2)$ \\
Cerebrovascular Disorders & $621(15.0)$ \\
Epilepsy & $490(11.8)$ \\
Substance-related Disorder &
\end{tabular}

Rates of exposure to each SGA and to the co-prescribed psychotropic medications are noted in Table 2.

Table 2. Prescribed Antipsychotic and Co-Prescribed Psychotropic Medications

\begin{tabular}{ll}
\hline Antipsychotic Medication & $\begin{array}{l}\text { N of Patients } \\
\text { Prescribed } \\
\text { Medication (\%) }\end{array}$ \\
\hline Aripiprazole & $601(14.5)$ \\
Ziprasidone & $597(14.4)$ \\
Quetiapine & $1201(29.0)$ \\
Olanzapine & $1206(29.1)$ \\
Risperidone & $3123(75.4)$ \\
Haloperidol or Fluphenazine & $188(4.5)$ \\
Prescribed Multiple SGAs & $1756(42.4)$ \\
Co-prescribed Psychotropic Medications & \\
SSRIs & $2367(57.2)$ \\
SNRIs & $2002(48.4)$ \\
Psychostimulants & $3170(76.6)$ \\
Mood Stabilizers & $1898(45.9)$ \\
\hline
\end{tabular}

Compared to "no exposure" to SGAs, the use of multiple SGAs, co-prescribed psychostimulants, and comorbid hypertension were associated with a significantly increased risk of arrhythmia events, whereas co-prescribed SNRI compounds were associated with a significantly decreased risk of arrhythmia events (see Table 3). An increased risk of having ischemic/myocardial events was significantly associated with the co-prescription of mood stabilizers or SSRIs, and the presence of comorbid hypertension and obesity (see Table 4), but not with the use of a specific SGA.
Table 3. Adjusted Risk Ratios for Incident Arrhythmia Events Related to SGAs, Comorbid Conditions, or Co-prescribed Medications

\begin{tabular}{lll}
\hline Parameter & aRisk Ratio & 95\% Confidence Intervals \\
\hline Female & 1.30 & $0.99-1.70$ \\
Age & 1.00 & $0.96-1.04$ \\
African American & 0.86 & $0.65-1.15$ \\
Other, non-white race & 1.22 & $0.88-1.69$ \\
Comorbid Hypertension & 2.23 & $1.28-3.89 *$ \\
Aripiprazole & 1.43 & $0.49-4.18$ \\
Ziprasidone & 0.79 & $0.27-2.26$ \\
Quetiapine & 1.46 & $0.82-2.61$ \\
Olanzapine & 0.92 & $0.44-1.89$ \\
Risperidone & 1.13 & $0.79-1.62$ \\
Multiple SGAs & 2.37 & $1.17-4.83^{*}$ \\
SNRIs/other & 0.59 & $0.35-0.99 *$ \\
Mood Stabilizer & 1.31 & $0.95-1.81$ \\
Psychostimulants & 1.37 & $1.03-1.81 *$ \\
\hline${ }^{*} \leq .05$ & &
\end{tabular}

Table 4. Adjusted Risk Ratios for Incident Ischemic/Myocardial Events Related to SGAs, Comorbid Conditions, or Co-prescribed Medications

\begin{tabular}{lll}
\hline Parameter & aRisk Ratio & $\begin{array}{l}\text { 95\% Confidence } \\
\text { Intervals }\end{array}$ \\
\hline Female & 1.28 & $0.87-1.89$ \\
Age & 1.00 & $0.94-1.05$ \\
African American & 1.18 & $0.78-1.79$ \\
Other, non-white race & 1.29 & $0.78-2.14$ \\
Comorbid Hypertension & 3.97 & $1.96-8.07^{*}$ \\
Comorbid Obesity/Overweight & 2.21 & $1.34-3.67^{*}$ \\
Aripiprazole & 1.30 & $0.18-9.16$ \\
Ziprasidone & 1.69 & $0.50-5.69$ \\
Quetiapine & 0.52 & $0.12-2.18$ \\
Olanzapine & 0.29 & $0.04-2.07$ \\
Risperidone & 1.00 & $0.60-1.66$ \\
Multiple SGAs & 1.17 & $0.43-3.20$ \\
SSRIs & 1.91 & $1.18-3.09 *$ \\
Mood Stabilizer & 1.68 & $1.06-2.68^{*}$ \\
Psychostimulants & 1.24 & $0.93-1.78$ \\
\hline * $p \leq .05$ & &
\end{tabular}

\section{Discussion}

In this population-based investigation, exposure to multiple SGAs and co-prescribed psychostimulants was associated with an increased risk of arrhythmia events, whereas co-prescribed SNRI compounds were associated with a significantly decreased risk of arrhythmia events. Although the number of RCTs involving pediatric patients with mental illness has increased over the past decade, no comparative RCTs have yet addressed the relative safety associated with individual antipsychotic agents, the co-prescription of two or more SGAs, or the co-prescription of multiple classes of psychotropic medications which were evident in this heterogeneous patient cohort. ${ }^{[28]}$ Based on the published safety profiles for agents in each class of medication, SGAs as a drug 
class are documented to prolong corrected QT intervals, ${ }^{[12]}$ but relatively few SGAs used as monotherapy prolong QT intervals at a rate known to be associated with subsequent cardiovascular adverse events. ${ }^{[6,28,29]}$ However, the risk attributable to individual SGAs appears to be increased when multiple SGAs are prescribed as polypharmacy and over prolonged periods.

Consistent with our results, psychostimulants have also previously been found to be associated with an increased incidence of arrhythmias. ${ }^{[13,14,30]}$ Moreover, the risk of developing ischemic/myocardial events was significantly associated with the co-prescription of mood stabilizers or SSRIs, but not with the use of SGAs which comports with some previous findings but not others. ${ }^{[5,10]}$ The protective effect of SNRI compounds has been previously identified ${ }^{[10]}$ as well and may be related to briefer periods of drug exposure or to a practice transition during the study period in which many pediatric patients were switched from SSRIs and to SNRIs. MSM analyses employing time-varying, monthly coding of medication exposure were sensitive to these medication changes and the resulting changes in documented adverse events. Finally, other factors bridging or mediating exposure to the psychotropic drugs evaluated and cardiovascular toxicity are also associated with cardiometabolic parameters (i.e., blood pressure, obesity). ${ }^{[7,8]}$ Comorbid hypertension was significantly associated with an increased risk for ischemic/myocardial events and for arrhythmias, whereas obesity/overweight was only associated with an increased risk of ischemic/myocardial events.

Comparing our MSM results with previous analyses using conventional logistic regression methods in this data set, substantial agreement exists regarding the negative effects of patient exposure to multiple antipsychotics, i.e., a significantly higher risk for incident cardiovascular events, incident obesity/weight gain, other signs of metabolic disruption (i.e., incident type 2 diabetes mellitus and dyslipidemia), and to mood stabilizers and SSRIs. ${ }^{[27]}$ However, the use of MSM procedures has further clarified the association between SGAs, especially in combination, and their dysrhythmia effects as mediated by comorbid hypertension and co-prescription of psychostimulants, as well as the lack of a systematic association between exposure to SGAs over time and incident ischemic/myocardial events/disorders.

The perspective provided by this observational, longitudinal database has several strengths. The cohort represents a large, heterogeneous group of children and adolescents with varying periods of SGA exposure ranging from brief treatment ( $<5$ months: $35 \%$ ) to long-term treatment ( 6 to 90 months: $65 \%)$. There is sufficient power in the treated cohort to detect

Published by Sciedu Press low-incidence cardiovascular events/conditions, and combine these conditions into related groupings for investigation. Previous studies have also found that although observational (Medicaid) databases provide much less detailed information on individuals, the physician diagnoses and utilization data are valid and more reliable than client or family selfreports. ${ }^{[31]}$ However, several limitations must also be kept in mind. No structured research and clinical interviews were employed to confirm any of the coded medical diagnoses, although independent investigations have been conducted to validate the psychiatric diagnoses and pharmacotherapy used. ${ }^{[24,25]}$ The reporting of non-psychiatric adverse events and comorbid cardio-metabolic or congenital conditions was based on reporting to or observation by a primary care physician and is, consequently, likely to be an under-estimate. These results report associations and, as a result, directions of causality cannot typically be inferred; however, MSM analyses are designed to better estimate "causal" associations by overcoming many sources of bias in non-randomized and controlled datasets. Key risk factors such as family history of obesity, metabolic disorders, and cardiovascular disorders were not available in the database and are not modeled in these analyses. Finally, although many significant covariates have been controlled for, other unmeasured differences in patients may have explained the findings.

\section{Conclusion}

In summary, MSM analyses have again demonstrated their utility for comparing multiple treatment effects, enhancing the results obtained from observational, longitudinal data sets, controlling for confounding variables, and yielding more precise estimates of treatment effects when randomized, controlled trials are not feasible.

\section{ACKNOWLEDGEMENTS}

Data acquisition was supported by a State Mental Health Data Infrastructure Grant (SAMHSA SM54192). Funding for these analyses was provided through a Clinical Incentive Research Grant from the University of South Carolina, Office of the Provost, which had no involvement in the conduct of the research or the reporting of these results. The views expressed do not necessarily represent those of the funding agency or official findings of the South Carolina Department of Health and Human Services (Medicaid).

\section{CONFLiCTS OF INTEREST Disclosure}

Dr. Tripathi has received funding from the American Heart Association. Dr. Black has no conflicts to disclose. Dr. Jerrell has served on advisory boards for Eli Lilly and Bristol Myers Squibb, and received research funding from NIH, Eli Lilly, and Bristol Myers Squibb. None of these entities 
had any involvement in or provided support for the research reported in this manuscript and the authors have no direct financial relationship with any of the commercial entities mentioned in this paper.

\section{REFERENCES}

[1] Burcu M, Zito JM, Ibe A, et al. Atypical antipsychotic use among Medicaid-insured children and adolescents: duration, safety, and monitoring implications. J Child Adolesc Psychopharmacol. 2014 24(3): 112-9. http://dx.doi.org/10.1089/cap. 2013.0094

[2] Cooper WO, Hickson GB, Fuchs C, et al. New users of antipsychotic medications among children enrolled in TennCare. Archives Pediatric Adolescent Medicine. 2004; 158(8): 753-9.

[3] Lohr WD, Chowning RT, Stevenson MD, et al. Trends in Atypical Antipsychotics Prescribed to Children Six Years of Age or Less on Medicaid in Kentucky. J Child Adolesc Psychopharmacol. 2015; 25(5): 440-3. http://dx.doi.org/10.1089/cap. 2014.0057

[4] Olfson M, Druss BG, Marcus SC. Trends in mental health care among children and adolescents. New England Journal Medicine. 2015; 372(21): 2029-38. http://dx.doi.org/10.1056/NEJMs a1413512

[5] Correll CU, Manu P, Olshanskiy V, et al. Cardiometabolic risk of second-generation antipsychotic medications during first-time use in children and adolescents. Journal American Medical Association. 2009; 302(16): 1765-73. http://dx.doi.org/10.1001/j ama. 2009.1549

[6] Ray WA, Chung CP, Murray KT, et al. Atypical antipsychotic drugs and the risk of sudden cardiac death. New England Journal Medicine. 2009; 360(3): 225-35. http://dx.doi.org/10.1056/NEJMoaO 806994

[7] Correll CU. Weight gain and metabolic effects of mood stabilizers and antipsychotics in pediatric bipolar disorder: a systematic review and pooled analysis of short-term trials. Journal American Academy Child Adolescent Psychiatry. 2007; 46(6): 687-700.

[8] Fedorowicz VJ, Fombonne E. Metabolic side effects of atypical antipsychotics in children: a literature review. Journal Psychopharmacology. 2005; 19(5): 533-50.

[9] Ellsworth AJ, Witt DM, Dugdale DC, et al. Mosby's Medical Drug Reference. St. Louis MO: Mosby. 2006.

[10] Taylor D, Lenox-Smith A, Bradley A. A review of the suitability of duloxetine and venlafaxine for use in patients with depression in primary care with a focus on cardiovascular safety, suicide and mortality due to antidepressant overdose. Ther Adv Psychopharmacol. 2013; 3(3): 151-61. http://dx.doi.org/10.1177/204512531 2472890

[11] Whang W, Kubzansky LD, Kawachi I, et al. Depression and risk of sudden cardiac death and coronary heart disease in women: results from the Nurses' Health Study. Journal American College Cardiology. 2009; 17; 53(11): 950-8. http://dx.doi .org/10.1016/j.jacc. 2008.10 .060

[12] Francis PD. Effects of psychotropic medications on the pediatric electrocardiogram and recommendations for monitoring. Current Opinion Pediatrics. 2002; 14(2): 224-30.

[13] Schelleman H, Bilker WB, Strom BL, et al. Cardiovascular events and death in children exposed and unexposed to ADHD agents. Pediatrics. 2011; 127(6): 1102-10. http://dx.doi.org/10.1542/p eds. 2010-3371

[14] Winterstein AG, Gerhard T, Kubilis P, et al. Cardiovascular safety of central nervous system stimulants in children and adolescents: population based cohort study. BMJ. 2012; 345: e4627. http: $/ / \mathrm{dx}$.doi.org/10.1136/bmj.e4627

[15] Keck PE. The role of second-generation antipsychotic monotherapy in the rapid control of acute bipolar mania. Journal Clinical Psychiatry. 2005; 66 (suppl.3): 5-11.

[16] Suarez D, Haro JM, Novick D, et al. Marginal structural models might overcome confounding when analyzing multiple treatment effects in observational studies. Journal Clinical Epidemiology. 2008; 61(6): 525-30. http://dx.doi.org/10.1016/j.jclinepi.20 07.11 .007

[17] Hernán MA, Hernández-Díaz S, Robins JM. A structural approach to selection bias. Epidemiology. 2004; 15(5): 615-25.

[18] Hernán MA, Brumback BA, Robins JM. Marginal structural models to estimate the joint causal effect of nonrandomized treatments. Journal American Statistical Association. 2001; 96(454): 440-8.

[19] Lambert M, Schimmelmann BG, Schacht A, et al. Differential 3year effects of first-versus second-generation antipsychotics on subjective well-being in schizophrenia using marginal structural models. Journal Clinical Psychopharmacology. 2011; 31(2): 226-30. http://dx.doi.org/10.1097/JCP.0b013e3182114d21

[20] Pirracchio R, Sprung CL, Payen D, et al. Utility of time-dependent inverse-probability-of-treatment weights to analyze observational cohorts in the intensive care unit. Journal Clinical Epidemiology. 2011; 64(12): 1373-82. http://dx.doi.org/10.1016/j.jclin epi.2011.02.009

[21] Suarez D, Borràs R, Basagaña X. Differences between marginal structural models and conventional models in their exposure effect estimates: a systematic review. Epidemiology. 2011; 22(4): 586-8. http://dx.doi.org/10.1097/EDE.0b013e31821d0507

[22] Faries DE, Ascher-Svanum H, Belger M. Analysis of treatment effectiveness in longitudinal observational data. Journal Biopharmaceutical Statistics. 2007; 17(5): 809-26.

[23] Robins JM, Hernán MA, Brumback B. Marginal structural models and causal inference in epidemiology. Epidemiology. 2000; 11(5): $550-60$.

[24] Jerrell JM. Pharmacotherapy in the community-based treatment of children with Bipolar I Disorder. Human Psychopharmacology Clinical Experimental. 2008; 23: 53-9.

[25] Jerrell JM, Shugart MA. Community-based care for youths with early and very-early onset bipolar I disorder. Bipolar Disorders. 2004; 6: 299-304.

[26] Curtis LH, Hammill BG, Eisenstein EL, et al. Using inverse probability-weighted estimators in comparative effectiveness analyses with observational databases. Medical Care. 2007; 45(10 Suppl 2): S103-S107.

[27] McIntyre RS, Jerrell JM. Metabolic and cardiovascular adverse events associated with antipsychotic treatment in children and adolescents. Archives Pediatric Adolescent Medicine. 2008; 162(10): 929-35. http://dx.doi.org/10.1001/archpedi.162.10.929

[28] De Hert M, Detraux J, van Winkel R, et al. Metabolic and cardiovascular adverse effects associated with antipsychotic drugs. Nat Rev Endocrinol. 2011; 8(2): 114-26. http://dx.doi.org/10.1038/n rendo.2011.156

[29] Darbà J, Kaskens L, Aranda P, et al. A simulation model to estimate 10-year risk of coronary heart disease events in patients with 
schizophrenia spectrum disorders treated with second-generation antipsychotic drugs. Annals Clinical Psychiatry. 2013; 25(1): 17-26.

[30] Cooper WO, Habel LA, Sox CM, et al. ADHD drugs and serious cardiovascular events in children and young adults. New England Journal Medicine. 2011; 365(20): 1896-904. http://dx.doi .org $/ 10.1056 /$ NEJMoa1110212
[31] Hennessy S, Leonard CE, Freeman CP, et al. Validation of diagnostic codes for outpatient-originating sudden cardiac death and ventricular arrhythmia in Medicaid and Medicare claims data. Pharmacoepidemiology Drug Safety. 2010; 19(6): 555-62. http://dx.doi.org/10. 1002/pds. 1869 